\title{
Interface entre exposição química e riscos à saúde da criança: Conhecimento de
}

\section{graduandos de Enfermagem}

Interface between chemical exposure and risks to children's health: Nursing undergraduate students' knowledge

Interfaz entre exposición química y riesgos para la salud infantil: Conocimiento de los graduados en Enfermería

Rayssa Bravo de Oliveira Vollmer ORCID: https://orcid.org/0000-0002-4882-7985

Universidade Federal Fluminense, Brasil E-mail: rayssa_vollmer@hotmail.com

Marcela de Abreu Moniz

ORCID: https://orcid.org/0000-0002-8481-7258

Universidade Federal Fluminense, Brasil

E-mail: marceladeabreumoniz@gmail.com

Joana de Andrade Nobre Ferraz

ORCID: https://orcid.org/0000-0002-7781-4593

Universidade Federal Fluminense, Brasil E-mail: joanaferraz.rc@ hotmail.com

Rayara Mozer Dias

ORCID: https://orcid.org/0000-0003-1170-0216

Universidade do Estado do Rio de Janeiro, Brasil

E-mail: rayaramozer@gmail.com

Lídia Santos Soares

ORCID: https://orcid.org/0000-0002-3170-8592 Universidade Federal Fluminense, Brasil E-mail: lidiasantossoares@gmail.com

Brunno Lessa Saldanha Xaxier

ORCID: https://orcid.org/0000-0002-7431-9108 Universidade Federal Fluminense, Brasil E-mail: brunnoprof@yahoo.com.br Jane Baptista Quitete

ORCID: https://orcid.org/0000-0003-0330-458X Universidade Federal Fluminense, Brasil

E-mail: janequitete@gmail.com

Rosana de Carvalho Castro

ORCID: https://orcid.org/0000-0003-0379-9244 Universidade Federal Fluminense, Brasil

E-mail: rcastro@id.uff.br

Maria da Anunciação Silva

ORCID: https://orcid.org/0000-0002-0069-5100 Universidade Federal Fluminense, Brasil

E-mail: inaiask8@terra.com.br

Yasmim Campos dos Santos Maia

ORCID: https://orcid.org/0000-0001-7816-701X

Universidade Federal Fluminense, Brasil

E-mail: yasmiimcampoos@gmail.com

Miriellen Bueno da Silva

ORCID: https://orcid.org/0000-0001-5353-2412

Universidade Federal Fluminense, Brasil

E-mail: mbdsbrasil@gmail.com

Ingrid da Silva Souza

ORCID: https://orcid.org/0000-0001-6675-0590

Universidade Federal Fluminense, Brasil

E-mail: ingridsilvasouza2105@gmail.com

\section{Resumo}

Introdução: $O$ avanço nas políticas de prevenção e pesquisas em saúde ambiental infantil exige a qualificação de profissionais de saúde com conhecimentos e habilidades sobre riscos à saúde da criança da exposição a contaminantes 
químicos ambientais. Objetivo: Avaliar se estudantes de enfermagem identificam a relação entre fatores de exposição química e riscos à saúde da criança e se este conhecimento difere conforme o ano de graduação. Método: Estudo transversal com questionário aplicado a 120 estudantes do curso de graduação em enfermagem da Universidade Federal Fluminense, campus Rio das Ostras, Rio de Janeiro, Brasil. A análise estatística envolveu o teste Quiquadrado, exato de Fisher e Kruskal-Wallis. Resultados: Evidenciou-se diferença significativa no conhecimento dos estudantes entre os cinco anos de graduação sobre os seguintes fatores de exposição: amamentação, uso de drogas durante a gestação e solo com a presença de pesticidas. Destaca-se que, um número maior de alunos do terceiro ano concordou com as assertivas sobre tais fatores. Apesar do conhecimento sobre metilmercúrio ter se diferenciado estatisticamente entre os estudantes dos cinco anos, 44,9\% não souberam opinar sobre este xenobiótico. Conclusão: Os participantes dos últimos anos do curso de graduação em enfermagem avaliado não demonstraram conhecimento adequado sobre alguns fatores de exposição química infantil indicando que o contexto formativo não tem ofertado atividades de ensino sobre este tema. Tal desconhecimento dos estudantes revela que a inclusão de conteúdos relativos à saúde ambiental infantil, especialmente da toxicologia, no currículo da enfermagem é fundamental.

Palavras-chave: Enfermagem; Saúde ambiental; Pediatria.

\begin{abstract}
Introduction: The advances in prevention politics and research on children's environmental health require the qualification of health professionals with knowledge and skills on the effects and risks of exposure of environmental chemical contaminants to children's health. Objective: Evaluate if nursing students were able to identify the relationship between the chemical exposure and the risks to children's health and if this knowledge is different based on each year of college. Method: Cross-sectional study with a questionnaire applied to 120 Nursing undergraduate students, from Federal Fluminense University, Rio das Ostras campus, Rio de Janeiro, Brazil. Statistical analysis involved the Chi-square test, Fisher's exact test, and Kruskal-Wallis test. Results: There was a significant difference in the students' knowledge among the undergraduate years about the effect of chemical exposure during breastfeeding, drug use during pregnancy, and exposure to soil with pesticides during childhood. The cohort with the highest comprehension of these effects was third-year students (in a five-year program. Not only the knowledge about methylmercury was different throughout the five years of the nursing course, but $44.9 \%$ weren't able to answer about this xenobiotic. Conclusion: Participants evaluated in their final year of study were unable to demonstrate appropriate knowledge of some factors of chemical exposure in children, which indicates that education on this theme insufficient. These results highlight the fundamental need for improvements in teaching related to children's environmental health, especially toxicology, in the nursing curriculum.
\end{abstract}

Keywords: Nursing; Environmental health; Pediatrics.

\title{
Resumen
}

Introducción: El avance en las políticas de prevención e investigación en salud ambiental infantil requiere la calificación de los profesionales de salud con conocimientos y habilidades a cerca de los riesgos para la salud de los niños la exposición a los contaminantes químicos ambientales. Objetivo: Evaluar si los estudiantes de enfermería identifican la relación entre los factores de exposición a sustancias químicas y los riesgos para la salud de los niños y si este conocimiento difiere según el año de graduación. Método: Estudio transversal con cuestionario aplicado a los 120 estudiantes de enfermería de la Universidad Federal Fluminense, campus Rio das Ostras, Rio de Janeiro, Brasil. El análisis estadístico involucró la prueba de Chi-cuadrado, la prueba exacta de Fisher y Kruskal-Wallis. Resultados: Se hizo evidente una diferencia significativa en el conocimiento de los estudiantes entre los cinco años de graduación a cerca de los siguientes factores de exposición: lactancia materna, uso de drogas durante el embarazo y suelo con presencia de pesticidas. Se hace importante destacar que, un mayor número de estudiantes del tercer año coincidieron con las afirmaciones sobre dichos factores. A pesar de que el conocimiento sobre el metilmercurio difiere estadísticamente entre los estudiantes de cinco años, el 44,9\% no sabía cómo opinar sobre este xenobiótico. Conclusión: Los participantes de los últimos años de la carrera de enfermería evaluados no demostraron un conocimiento adecuado sobre algunos factores de la exposición química infantil, lo que indica que el contexto de formación no ha ofrecido actividades docentes sobre esta temática. Tal desconocimiento por parte de los estudiantes revela que la inclusión de contenidos relacionados a la salud ambiental infantil, especialmente la toxicología, en el currículo de enfermería es fundamental.

Palabras clave: Enfermería; Salud ambiental; Pediatría.

\section{Introdução}

A exposição química ambiental está diretamente relacionada ao desenvolvimento de doenças em todas as fases da vida humana. A infância se constitui em um uma fase especialmente vulnerável à exposição aos diversos contaminantes ambientais, pois, podem produzir impactos à saúde infantil de grande magnitude. Cerca de três milhões de crianças menores de 
cinco anos de idade morrem a cada ano devido a doenças relacionadas a poluentes ambientais, uma vez que, nesta faixa etária a influência dos fatores ambientais sobre o processo saúde-doença é mais relevante que o fator genético (Perloth \& Branco, 2017).

A poluição do ar é uma das maiores ameaças à saúde da criança, sendo responsável por uma das principais causas de mortalidade infantil no mundo. A Organização Mundial de Saúde (OMS) estima que 97\% das cidades de países de baixa e média renda não respeitam os parâmetros recomendados para qualidade do ar. (Jan, Magoon \& Ross, 2019).

Um estudo brasileiro revelou que as principais causas associadas à taxa de mortalidade infantil se devem à ausência de acesso aos serviços de saneamento ambiental e saúde. Os fatores socioambientais são determinantes das condições de saúde e morbimortalidade infantil (Sousa, Campos, Silva, Bezerra \& Lira, 2016).

Considerando que o organismo infantil ainda se encontra em fase precoce com vários tecidos e órgãos em processo de desenvolvimento e maturação, a persistência sob condições adversas, tais como a exposição a substâncias tóxicas, pode causar danos temporários ou permanentes com ressonância na condição de saúde atual e futura. Algumas características fisiológicas da criança contribuem para elevar o risco de intoxicação aguda e crônica, devido a maior demanda de água e alimentos, menor peso corporal e outras condições de metabolismo e distribuição das substâncias químicas no organismo que são diferenciadas do organismo do adulto (Perloth \& Branco, 2017; Mazoto, Filhote, Câmara \& Asmus, 2011).

Outro agravante refere-se ao comportamento infantil, que eleva a vulnerabilidade das crianças à exposição química ambiental, tais como o ato de engatinhar, colocar mãos e objetos na boca, brincar próximo ao solo. Desse modo, a exposição infantil aos contaminantes químicos pode ocorrer pelas vias: vertical (intraútero e amamentação) e ambiental. A exposição química via vertical ou na primeira infância pode levar a redução da função pulmonar, transtornos comportamentais, baixo peso ao nascer, câncer infantil, aumento do número de casos de doenças cardíacas e outros problemas que poderão ser irreversíveis (Mazoto et al., 2011).

Frente ao exposto, as noções de toxicologia na saúde maternoinfantil são de extrema importância para os profissionais enfermeiros, que possuem um papel fundamental na realização de ações de promoção da saúde, vigilância e gerenciamento de riscos químicos, educação popular e educação permanente em saúde ambiental. (Moniz, Sabóia, Daher \& Pereira, 2017). Nesse contexto, este estudo teve o objetivo de avaliar se estudantes de enfermagem identificam a relação entre fatores de exposição química e riscos à saúde da criança e se este conhecimento difere conforme o ano de graduação.

\section{Metodologia}

Um estudo transversal, observacional, descritivo de natureza quantitativa foi conduzido por meio de autopreenchimento de questionários por graduandos de enfermagem da Universidade Federal Fluminense, campus Rio das Ostras entre os meses de abril a julho de 2017. O presente estudo segue a tendência de uso da abordagem quantitativa em pesquisas no campo da avaliação educacional (Pereira \& Ortigão, 2016).

A amostra do estudo incluiu 120 estudantes de enfermagem do primeiro ao quinto ano do curso de graduação em Enfermagem. Partiu-se de um censo com registro de 187 alunos matriculados em disciplinas do curso e houve perda menor que 5\% por ausência de captação e/ou recusa de participação. Os critérios de inclusão foram: ser estudante com matrícula ativa do primeiro ao décimo período do curso de graduação em Enfermagem, campus Rio das Ostras da Universidade Federal Fluminense; e ter idade a partir dos 18 anos.

O questionário foi construído com questões avaliativas de conhecimento sobre fatores de exposição química ambiental e vertical que produzem riscos à saúde de adultos e crianças. Nesse artigo, detalham-se as seguintes dimensões e as respectivas variáveis ao tema de estudo: dados sociodemográficos (sexo, faixa etária, ano de graduação), sendo o ano de 
graduação como variável ordinal dependente; conhecimento acerca da relação existente entre fatores de exposição química durante a gestação (variáveis independentes: contato materno durante a gestação com pesticidas, contato materno durante a gestação com outras substâncias químicas (benzeno, metais pesados) por meio de tinta no cabelo, contato materno durante a gestação com nicotina, contato materno durante a gestação com maconha, ambiente com presença de gases tóxicos, uso de drogas durante a gestação, contato materno durante a gestação com álcool, e contato materno durante a gestação com medicamentos); conhecimento acerca da relação entre fatores de exposição química durante a infância e riscos à saúde da criança (variáveis independentes: contato com cosméticos, água ou alimento com a presença de bisfenol, contato com inseticidas e outros praguicidas utilizados na casa e redondezas, solo com a presença de pesticidas, amamentação, e água ou alimento com a presença de estrogênios); conhecimento sobre os efeitos à saúde da criança e algumas vias de exposição ambiental ao dióxido de enxofre, metilmercúrio e chumbo (variáveis independentes: o dióxido de enxofre é um dos causadores da asma infantil e pode ser inalado pela fumaça do cigarro, queima de biomassa ou fumaça de veículos; o metilmercúrio é uma categoria química que pode estar presente na carne do peixe e pode produzir efeitos de neurotoxicidade infantil; e o chumbo é neurotóxico, nefrotóxico e hematotóxico e pode estar presente em tintas, baterias, pilhas, baterias, tinturas para cabelo e cosméticos). Foram selecionados estes três xenobióticos por caracterizarem-se em agentes tóxicos com bastante fundamentação em literatura internacional pediátrica (Perloth \& Branco, 2017; Mazoto et al., 2011).

Os dados foram tabulados com dupla digitação e analisados utilizando o software estatístico Statistical Package for the Social Sciences, versão 21.0., aplicando-se os testes de qui-quadrado e de Kruskal-Wallis, com valor descritivo final de $\mathrm{p}<0,05$.

Este estudo é parte integrante do projeto intitulado "Educação ambiental e enfermagem: caminho para a Ética, a Sustentabilidade e a Promoção da Saúde", que foi aprovado pelo Comitê de Ética em Pesquisa da Universidade Federal Fluminense, em fevereiro de 2017 sob o número de parecer 1.934.809.

\section{Resultados}

Do universo amostral de 120 participantes do estudo, 87\% eram do sexo feminino, enquanto $13 \%$ do sexo masculino. Em relação à faixa etária, $64 \%$ das pessoas tinham acima de 20 anos, sendo que a variação média da idade dos participantes foi de 22 anos (desvio-padrão 5,09). Sobre o ano de graduação, observou-se que, 20\% dos respondentes estavam no primeiro ano, $25 \%$ no segundo ano, $24 \%$ no terceiro ano, $17 \%$ no quarto ano e $14 \%$ no quinto ano. Os dados pressupõem que à medida que os estudantes avancem nos períodos do curso de graduação, o número de alunos por período e ano diminua.

No que se refere aos riscos químicos à saúde maternoinfantil, observou-se que, houve diferença significativa no conhecimento dos participantes entre os cinco anos de graduação apenas sobre o uso de drogas durante a gestação $(p=0,008)$ (Tabela 1). Nesta variável, a totalidade dos alunos do terceiro ano de graduação concordou com esse risco à saúde fetal. Para as demais variáveis, não houve diferenças significativas.

Assim, notou-se que a grande maioria dos estudantes, independente do ano de graduação, concordou que o contato materno com nicotina, álcool, maconha, gases tóxicos, pesticidas e substâncias químicas presente em tintas de cabelo pode se caracterizar em possíveis fatores de exposição química fetal.

Em relação à exposição da gestante aos medicamentos e riscos à saúde fetal, identificou-se conhecimento insuficiente de 38,3\% dos estudantes, independente do ano de graduação. 
Tabela 1. Conhecimento dos estudantes sobre a relação entre os fatores de exposição química gestacional e os riscos à saúde da criança, segundo o ano de graduação.

\begin{tabular}{|c|c|c|c|c|c|c|c|c|c|c|c|c|}
\hline \multirow{4}{*}{ Variável } & \multicolumn{10}{|c|}{ Ano de graduação } & \multirow{4}{*}{$\begin{array}{l}\text { Total } \\
\text { N(\%) }\end{array}$} & \multirow{4}{*}{ Valor $\mathbf{p}$} \\
\hline & \multicolumn{2}{|c|}{1} & \multicolumn{2}{|c|}{2} & \multicolumn{2}{|c|}{3} & \multicolumn{2}{|c|}{4} & \multicolumn{2}{|c|}{5} & & \\
\hline & SIM & NÃO & SIM & NÃO & SIM & NÃO & SIM & NÃO & SIM & NÃO & & \\
\hline & $\mathbf{N}(\%)$ & $\mathbf{N}(\%)$ & $\mathbf{N}(\%)$ & $\mathbf{N}(\%)$ & $\mathbf{N}(\%)$ & $\mathbf{N}(\%)$ & $\mathbf{N}(\%)$ & $\mathbf{N}(\%)$ & $\mathbf{N}(\%)$ & $\mathbf{N}(\%)$ & & \\
\hline $\begin{array}{c}\text { Contato } \\
\text { materno } \\
\text { durante a } \\
\text { gestação com } \\
\text { pesticidas }\end{array}$ & $17(14,2)$ & $7(5,8)$ & $26(21,7)$ & $4(3,3)$ & $26(21,7)$ & $3(2,5)$ & $19(15,8)$ & $1(0,8)$ & $16(13,3)$ & $1(0,8)$ & $120(100)$ & 0,114 \\
\hline $\begin{array}{c}\text { Contato } \\
\text { materno } \\
\text { durante a } \\
\text { gestação com } \\
\text { outras } \\
\text { substâncias } \\
\text { químicas } \\
\text { (benzeno, } \\
\text { metais } \\
\text { pesados) por } \\
\text { meio de tinta } \\
\text { no cabelo } \\
\end{array}$ & $20(16,7)$ & $4(3,3)$ & $28(23,3)$ & $2(1,7)$ & $26(21,7)$ & $3(2,5)$ & $20(16,7)$ & $0(0,0)$ & $14(11,7)$ & $3(2,5)$ & $120(100)$ & 0,299 \\
\hline $\begin{array}{c}\text { Contato } \\
\text { materno } \\
\text { durante a } \\
\text { gestação com } \\
\text { nicotina }\end{array}$ & $23(19,2)$ & $1(0,8)$ & $25(20,8)$ & $5(4,2)$ & $26(21,7)$ & $3(2,5)$ & $19(15,8)$ & $1(0,8)$ & $16(13,3)$ & $1(0,8)$ & $120(100)$ & 0,488 \\
\hline $\begin{array}{c}\text { Contato } \\
\text { materno } \\
\text { durante a } \\
\text { gestação com } \\
\text { maconha }\end{array}$ & $21(17,5)$ & $3(2,5)$ & $24(20,0)$ & $6(5,0)$ & $23(19,2)$ & $6(5,0)$ & $18(15,0)$ & $2(1,7)$ & $15(12,5)$ & $2(1,7)$ & $120(100)$ & 0,768 \\
\hline $\begin{array}{c}\text { Ambiente } \\
\text { com presença } \\
\text { de gases } \\
\text { tóxicos }\end{array}$ & $23(19,2)$ & $1(0,8)$ & $29(24,2)$ & $1(0,8)$ & $28(23,3)$ & $1(0,8)$ & $19(15,8)$ & $1(0,8)$ & $17(14,2)$ & $0(0,0)$ & $120(100)$ & 0,937 \\
\hline $\begin{array}{c}\text { Uso de drogas } \\
\text { durante a } \\
\text { gestação }\end{array}$ & $23(19,2)$ & $1(0,8)$ & $22(18,3)$ & $8(6,7)$ & $29(24,2)$ & $0(0,0)$ & $18(15,0)$ & $2(1,7)$ & $16(13,3)$ & $1(0,8)$ & $120(100)$ & 0,008 \\
\hline $\begin{array}{c}\text { Contato } \\
\text { materno } \\
\text { durante a } \\
\text { gestação com } \\
\text { álcool } \\
\end{array}$ & $21(17,5)$ & $3(2,5)$ & $24(20,0)$ & $6(5,0)$ & $27(22,5)$ & $2(1,7)$ & $19(15,8)$ & $1(0,8)$ & $15(12,5)$ & $2(1,7)$ & $120(100)$ & 0,472 \\
\hline $\begin{array}{c}\text { Contato } \\
\text { materno } \\
\text { durante a } \\
\text { gestação com } \\
\text { medicamentos }\end{array}$ & $11(9,2)$ & $13(10,8)$ & $19(15,8)$ & $11(9,2)$ & $19(15,8)$ & $10(8,3)$ & $14(11,7)$ & $6(5,0)$ & $11(9,2)$ & $6(5,0)$ & $120(100)$ & 0,491 \\
\hline
\end{tabular}

Fonte: Autores.

A Tabela 2 apresenta o conhecimento dos alunos dos cinco anos de graduação em relação às variáveis sobre a relação entre os fatores de exposição química durante a infância e os riscos à saúde da criança. Notaram-se evidências de diferenças significativas do conhecimento dos estudantes entre os cinco anos de graduação sobre a presença de pesticidas no solo $(\mathrm{p}=0,007)$ ser um fator de exposição que pode produzir riscos a saúde da criança. A maioria dos estudantes que considerou este fator era do terceiro ano do curso de graduação $(17,5 \%)$.

O último fator em que o conhecimento dos graduandos se diferenciou, de forma significativa, apesar de bordeline, foi amamentação ( $\mathrm{p}=0,050)$ (Tabela 2$)$, destacando-se que um percentual $(93,3 \%)$ expressivo de participantes, de todos os anos de 
graduação, que não reconheceu a amamentação como um possível fator de exposição química infantil, que nenhum estudante do segundo e do quarto ano e 82,3\% dos participantes do quinto ano não perceberam esta possível relação causa-efeito. Para as demais variáveis não houve diferença significativa.

Tabela 2. Conhecimento de estudantes de enfermagem sobre a relação entre os fatores de exposição química ambiental durante a infância e os riscos à saúde da criança, segundo o ano de graduação.

\begin{tabular}{|c|c|c|c|c|c|c|c|c|c|c|c|c|}
\hline \multirow{4}{*}{ Variável } & \multicolumn{12}{|c|}{ Ano de graduação } \\
\hline & \multicolumn{2}{|c|}{1} & \multicolumn{2}{|c|}{2} & \multicolumn{2}{|c|}{3} & \multicolumn{2}{|c|}{4} & \multicolumn{2}{|c|}{5} & \multirow{3}{*}{$\begin{array}{l}\text { Total } \\
\mathrm{N}(\%)\end{array}$} & \multirow{3}{*}{ Valor $\mathbf{p}$} \\
\hline & SIM & NÃO & SIM & NÃO & SIM & NÃO & SIM & NÃO & SIM & NÃO & & \\
\hline & $\mathbf{N}(\%)$ & $\mathbf{N}(\%)$ & $\mathbf{N}(\%)$ & $\mathbf{N}(\%)$ & $\mathbf{N}(\%)$ & $\mathbf{N}(\%)$ & $\mathbf{N}(\%)$ & $\mathbf{N}(\%)$ & $\mathbf{N}(\%)$ & $\mathbf{N}(\%)$ & & \\
\hline $\begin{array}{l}\text { Contato com } \\
\text { cosméticos }\end{array}$ & $5(4,2)$ & $19(15,8)$ & $8(6,7)$ & $22(18,3)$ & $9(7,5)$ & $20(16,7)$ & $7(5,8)$ & $13(10,8)$ & $6(5,0)$ & $11(9,2)$ & $120(100)$ & 0,812 \\
\hline $\begin{array}{c}\text { Água ou } \\
\text { alimento com } \\
\text { a presença de } \\
\text { bisfenol }\end{array}$ & $11(9,2)$ & $13(10,8)$ & $14(11,7)$ & $16(13,3)$ & $15(12,5)$ & $14(11,7)$ & $14(11,7)$ & $6(5,0)$ & $14(11,7)$ & $3(2,5)$ & $120(100)$ & 0,07 \\
\hline $\begin{array}{l}\text { Contato com } \\
\text { inseticidas e } \\
\text { outros } \\
\text { praguicidas } \\
\text { utilizados na } \\
\text { casa e } \\
\text { redondezas }\end{array}$ & $16(13,3)$ & $8(6,7)$ & $20(16,7)$ & $10(8,3)$ & $24(20,0)$ & $5(4,2)$ & $19(15,8)$ & $1(0,8)$ & $13(10,8)$ & $4(3,3)$ & $120(100)$ & 0,117 \\
\hline $\begin{array}{c}\text { Solo com a } \\
\text { presença de } \\
\text { pesticidas }\end{array}$ & $13(10,8)$ & $11(9,2)$ & $17(14,2)$ & $13(10,8)$ & $21(17,5)$ & $8(6,7)$ & $18(15,0)$ & $2(1,7)$ & $16(13,3)$ & $1(0,8)$ & $120(100)$ & 0,007 \\
\hline Amamentação & $1(0,8)$ & $23(19,2)$ & $0(0,0)$ & $30(25,0)$ & $4(3,3)$ & $25(20,8)$ & $0(0,0)$ & $20(16,7)$ & $3(2,5)$ & $14(11,7)$ & $120(100)$ & 0,05 \\
\hline $\begin{array}{c}\text { Água ou } \\
\text { alimento com } \\
\text { a presença de } \\
\text { estrogênios }\end{array}$ & $13(10,8)$ & $11(9,2)$ & $15(12,5)$ & $15(12,5)$ & $18(15,0)$ & $11(9,2)$ & $14(11,7)$ & $6(5,0)$ & $9(7,5)$ & $8(6,7)$ & $120(100)$ & 0,65 \\
\hline
\end{tabular}

Fonte: Autores.

Na Tabela 3, são apresentadas três afirmativas que se referem ao conhecimento sobre as substâncias químicas: dióxido de enxofre, metilmercúrio e chumbo, algumas de suas principais vias de exposição e alguns de seus efeitos tóxicos à saúde da criança. Percebeu-se que, apenas, o conhecimento sobre metilmercúrio se apresentou com diferença estatisticamente significativa $(\mathrm{p}=0,03)$ entre os estudantes dos cinco anos de graduação. Somente $12,7 \%$ dos participantes afirmaram concordar totalmente e $34,7 \%$ concordaram com esta afirmativa. Destaca-se, ainda, que os estudantes do terceiro ano (11,9\%) e quarto ano $(9,3 \%)$ foram os que mais concordaram com tal afirmativa e que, quase metade dos alunos (44,9\%) não soube opinar sobre o metilmercúrio. As afirmativas referentes ao dióxido de enxofre e ao chumbo não apresentaram diferenças significativas entre os anos de graduação. 
Tabela 3. Conhecimento de estudantes de enfermagem sobre os efeitos à saúde da criança e algumas vias de exposição ambiental ao dióxido de enxofre, metilmercúrio e chumbo, segundo o ano de graduação.

\begin{tabular}{|c|c|c|c|c|c|c|c|c|}
\hline \multirow[b]{2}{*}{ Variável } & \multirow[b]{2}{*}{$\begin{array}{l}\text { Escala de } \\
\text { resposta }\end{array}$} & \multicolumn{7}{|c|}{ Ano de graduação } \\
\hline & & $\begin{array}{c}1 \\
\mathbf{N}(\%)\end{array}$ & $\begin{array}{c}2 \\
\mathrm{~N}(\%)\end{array}$ & $\begin{array}{c}3 \\
\mathbf{N}(\%)\end{array}$ & $\begin{array}{c}4 \\
\mathbf{N}(\%)\end{array}$ & $\begin{array}{c}5 \\
\mathrm{~N}(\%)\end{array}$ & $\begin{array}{l}\text { Total } \\
\text { N(\%) }\end{array}$ & Valor $\mathbf{p}$ \\
\hline \multirow{6}{*}{$\begin{array}{c}\text { O dióxido de } \\
\text { enxofre é um } \\
\text { dos causadores } \\
\text { da asma infantil } \\
\text { e pode ser } \\
\text { inalado pela } \\
\text { fumaça do } \\
\text { cigarro, queima } \\
\text { de biomassa ou } \\
\text { fumaça de } \\
\text { veículos. }\end{array}$} & Concordo & $6(5,1)$ & $1(0,8)$ & $6(5,1)$ & $2(1,7)$ & $4(3,4)$ & $19(16,1)$ & \multirow{6}{*}{0,269} \\
\hline & Concordo & $13(11)$ & $16(13,6)$ & $10(8,5)$ & $12(10,2)$ & $5(4,2)$ & $56(47,5)$ & \\
\hline & Discordo & $1(0,8)$ & $2(1,7)$ & $0(0,0)$ & $0(0,0)$ & $1(0,8)$ & $4(3,4)$ & \\
\hline & $\begin{array}{c}\text { Discordo } \\
\text { totalmente }\end{array}$ & $1(0,8)$ & $2(1,7)$ & $0(0,0)$ & $0(0,0)$ & $0(0,0)$ & $3(2,5)$ & \\
\hline & $\begin{array}{l}\text { Não tenho } \\
\text { opinião }\end{array}$ & $3(2,5)$ & $9(7,6)$ & $12(10,2)$ & $5(4,2)$ & $7(5,9)$ & $36(30,5)$ & \\
\hline & Total & $24(20,3)$ & $30(25,4)$ & $28(23,7)$ & $19(16,1)$ & $17(14,4)$ & $118(100)$ & \\
\hline \multirow{6}{*}{$\begin{array}{c}\text { O } \\
\text { metilmercúrio é } \\
\text { uma categoria } \\
\text { química que } \\
\text { pode estar } \\
\text { presente na } \\
\text { carne do peixe e } \\
\text { pode produzir } \\
\text { efeitos de } \\
\text { neurotoxicidade } \\
\text { infantil. }\end{array}$} & $\begin{array}{l}\text { Concordo } \\
\text { totalmente }\end{array}$ & $1(0,8)$ & $5(4,2)$ & $5(4,2)$ & $1(0,8)$ & $3(2,5)$ & $15(12,7)$ & \multirow{6}{*}{0,033} \\
\hline & Concordo & $5(4,2)$ & $6(5,1)$ & $14(11,9)$ & $11(9,3)$ & $5(4,2)$ & $41(34,7)$ & \\
\hline & Discordo & $4(3,4)$ & $2(1,7)$ & $1(0,8)$ & $0(0,0)$ & $1(0,8)$ & $8(6,8)$ & \\
\hline & $\begin{array}{l}\text { Discordo } \\
\text { totalmente }\end{array}$ & $0(0,0)$ & $0(0,0)$ & $1(0,8)$ & $0(0,0)$ & $0(0,0)$ & $1(0,8)$ & \\
\hline & $\begin{array}{l}\text { Não tenho } \\
\text { opinião }\end{array}$ & $14(11,9)$ & $17(14,4)$ & $7(5,9)$ & $7(5,9)$ & $8(6,8)$ & $53(44,9)$ & \\
\hline & Total & $24(20,3)$ & $30(25,4)$ & $28(23,7)$ & $19(16,1)$ & $17(14,4)$ & $118(100)$ & \\
\hline \multirow{6}{*}{$\begin{array}{l}\text { O chumbo é } \\
\text { neurotóxico, } \\
\text { nefrotóxico e } \\
\text { hematotóxico e } \\
\text { pode estar } \\
\text { presente em } \\
\text { tintas, baterias, } \\
\text { pilhas, baterias, } \\
\text { tinturas para } \\
\text { cabelo e } \\
\text { cosméticos. }\end{array}$} & $\begin{array}{l}\text { Concordo } \\
\text { totalmente }\end{array}$ & $11(9,3)$ & $10(8,5)$ & $14(11,9)$ & $11(9,3)$ & $11(9,3)$ & $57(48,3)$ & \multirow{6}{*}{0,128} \\
\hline & Concordo & $10(8,5)$ & $14(11,9)$ & $12(10,2)$ & $7(5,9)$ & $5(4,2)$ & $48(40,7)$ & \\
\hline & Discordo & $1(0,8)$ & $2(1,7)$ & $0(0,0)$ & $0(0,0)$ & $0(0,0)$ & $3(2,5)$ & \\
\hline & $\begin{array}{c}\text { Discordo } \\
\text { totalmente }\end{array}$ & $0(0,0)$ & $0(0,0)$ & $0(0,0)$ & $0(0,0)$ & $0(0,0)$ & $0(0,0)$ & \\
\hline & $\begin{array}{l}\text { Não tenho } \\
\text { opinião }\end{array}$ & $2(1,7)$ & $4(3,4)$ & $2(1,7)$ & $1(0,8)$ & $1(0,8)$ & $10(8,5)$ & \\
\hline & Total & $24(20,3)$ & $30(25,4)$ & $28(23,7)$ & $19(16,1)$ & $17(14,4)$ & $118(100)$ & \\
\hline
\end{tabular}

Fonte: Autores.

\section{Discussão}

Os achados do presente estudo revelaram que a maior parte dos estudantes percebeu sobre a possibilidade de existirem riscos à saúde da criança em decorrência da exposição materna às drogas, embora não tenha sido explorado o nível de conhecimento dos estudantes sobre os tipos de efeitos tóxicos à saúde do feto, do neonato e da criança provocados por cada tipo de droga.

O consumo de substâncias psicoativas por gestantes e mulheres que amamentam pode produzir riscos severos em curto e em longo prazo à saúde da criança. O consumo do álcool durante a gestação pode ocasionar lesões orgânicas e neurológicas fetais, inclusive a síndrome alcoólica fetal, que é reconhecida como a maior causa de retardo mental no Ocidente, enquanto, o consumo do tabaco pela gestante pode aumentar o risco em $70 \%$ de sofrer aborto espontâneo, $40 \%$ de parto prematuro e nascimento de recém-nascido de baixo risco. Sobre as drogas ilícitas mais consumidas em todo o mundo, a maconha pode causar prematuridade, baixo peso ao nascer e diminuição do perímetro cefálico no feto e a cocaína/ crack pode provocar retardo no desenvolvimento e até óbito fetal (Rocha et al., 2016). 
O conhecimento dos estudantes sobre a exposição química a pesticidas, substâncias tóxicas presentes em tintura de cabelo e gases tóxicos na gestação enquanto fator de risco à saúde da criança também foi satisfatório. Diversos fatores de exposição química materna e agentes infecciosos podem colocar em risco a saúde do feto e, igualmente, do binômio mãe-bebê. Agrotóxicos e metais pesados, como mercúrio e metilmercúrio, conseguem atravessar a camada endotelial e afetar o tecido encefálico porque a barreira hematoencefálica não está completamente desenvolvida até os seis meses de idade. Condições neonatais, oriundas de exposição a fatores ambientais durante a gestação, o nascimento e o período pós-natal levam a mais de um milhão de mortes por ano (Zendron, 2015; Costa, Araujo Júnior, Lima \& Costa, 2014; Maldaner, Santos, Weinert \& Weinert, 2017).

Finkle, Battisti e Anastácio (2019) avaliaram estudos relacionados à exposição de agrotóxicos a crianças em propriedades rurais feitas entre os anos de 2014 a 2019 e os resultados apontaram possíveis danos à saúde, tais como, malformações congênitas, leucemia, efeitos neurológicos, asma, rinite alérgica.

O contato da gestante com as substâncias tóxicas presentes na tinta do cabelo, assim como o benzeno, pode causar risco de leucemia infantil. A tinta de cabelo, assim como alguns outros produtos cosméticos, não é recomendada às gestantes e lactantes por conterem substâncias prejudiciais à saúde do binômio mãe-bebê. A tintura de cabelo contém altas concentrações de aminas primárias que podem ser absorvidas no organismo rapidamente, gerando efeitos cancerígenos que podem originar células cancerígenas no bebê ainda intraútero. (Ferreira, Couto, Alves, Oliveira \& Koifman, 2012; Borson, Martins, Comune \& Guidi Junior, 2019).

Evidenciou-se, ainda, que 38,3\% dos estudantes, independente do ano de graduação, apresentou conhecimento inadequado acerca da exposição gestacional a medicamentos e a possibilidade de riscos à saúde da criança. Tal achado sugere que este tema seja pouco abordado durante a formação ou as estratégias e os espaços de ensino utilizados estejam sendo pouco eficazes. Medicamentos são potenciais teratogênicos, que são influenciados por inúmeros fatores como momento da gestação, via de administração, substâncias concomitantes, entre outros. Um estudo mostrou que os medicamentos mais utilizados por mulheres de regiões norte e ocidental da Europa que estão associados a risco materno-fetal são codeína, ibuprofeno e metoclopramida. Ainda assim, medicações com potencial teratogênico são utilizadas durante a gestação, pela ausência de alternativas recomendadas (Bernardes, 2018).

A amamentação é outro importante fator de exposição química infantil, e os achados desse estudo revelaram um conhecimento inadequado desta entre os estudantes participantes de todos os anos de graduação. O leite materno fornece nutrientes ao recém-nascido, bem como possui vantagens no âmbito de fatores imunológicos, cognitivos, psicoafetivos, econômicos e sociais, todavia, pode fornecer, também, ao bebê diversas substâncias tóxicas como dioxinas, mercúrio, nicotina, pesticidas, entre outras, as quais estão circulando no organismo materno (Perloth \& Branco, 2017).

Sobre os riscos de exposição química durante a infância, verificou-se que, o conhecimento dos graduandos sobre o contato da criança com pesticidas no solo ser um fator de risco à saúde se diferenciou entre os anos de graduação e que, 29,2\% não identificaram este tipo de risco. Existem, aproximadamente, 15 mil compostos químicos produzidos em todo o mundo, incluindo substâncias neurotóxicas, tais como, chumbo, solventes, mercúrio e pesticidas que causam riscos de intoxicação aguda e crônica em crianças. A via digestiva é uma das principais vias de exposição infantil aos agrotóxicos. Há evidências da interferência destes compostos nas ações estrogênicas e androgênicas, inibição da síntese de hormônios tireoidianos e efeitos imunotóxicos e neurotóxicos em crianças devida à exposição a estes poluentes químicos. Um estudo apontou ainda que quando se trata de exposição ambiental infantil, os agrotóxicos estão entre os xenobióticos de maior preocupação, e que mesmo considerando o uso intenso de agrotóxicos no Brasil, ainda há poucos estudos na literatura sobre os efeitos da exposição a agrotóxicos na saúde de crianças brasileiras (Mazoto et al., 2011; Moniz \& Vollmer, 2017; Nascimento, 2017). 
Por fim, apenas, o conhecimento dos participantes sobre o metilmercúrio apresentou relação com o ano de graduação. Boas partes dos estudantes não souberam opinar sobre os xenobióticos: metilmercúrio e o dióxido de enxofre. Este fato possivelmente se deve à ausência de inclusão de conteúdos relativos à toxicologia infantil e de saúde ambiental infantil de forma transversal nas disciplinas do currículo de graduação em Enfermagem. Alguns estudos revelaram conhecimento inadequado ou insuficiente de graduandos de enfermagem sobre a interface entre problemas ambientais e a saúde pública e que, questões contemporâneas ambientais, incluindo as condições de riscos de exposição química ambiental, e sustentabilidade devem ser contempladas na formação do enfermeiro (Moniz, Pereira, \& Marques, 2017; García, Nieto, Hidalgo, Martos \& Medina, 2018).

A poluição do ar é uma das maiores ameaças à saúde global e causa a morte de sete milhões de pessoas anualmente. Um dos poluentes atmosféricos é o dióxido de enxofre, que é o subproduto da queima de combustíveis fósseis e responsável pelo aumento da acidez da chuva, além de ser um dos principais causadores da asma infantil. Além deste, a contaminação do meio ambiente por agentes químicos resultantes de atividades industriais, produção agrícola e exploração mineral está relacionada ao aumento de malformações congênitas, câncer, distúrbios neurológicos e respiratórios da criança (WHO, 2017; Moniz \& Vollmer, 2017).

Um estudo mostrou a relação de fatores de exposição ambiental, com internação por razões respiratórias de crianças no estado de São Paulo. (Moraes, Almendra, Santana \& Galvani, 2019). Evidências de um estudo epidemiológico indicaram que exposições a poluentes atmosféricos, como o material particulado e óxidos de nitrogênio, estavam associadas a aumentos na taxa de mortalidade respiratória. Os efeitos da exposição de material particulado sobre o aparelho respiratório e cardiovascular podem estar relacionados ao aparecimento e agravamento de manifestações clínicas, em especial em populações vulneráveis como crianças e idosos (Gouveia, Leon, Junger, Lins \& Freitas, 2019).

Os metais pesados são substâncias xenobióticas, quimicamente muito reativas e bioacumulativas, que não são eliminadas pelo organismo de forma eficaz e rápida. O chumbo, o mercúrio e o cádmio são metais que podem estar presentes na água e em alimentos tais como o peixe e, assim, a principal porta de entrada desta substância no organismo é a digestiva. Já o metilmercúrio tem uma enorme absorção em nível gastrointestinal, sendo que a absorção do mercúrio orgânico é de $15 \%$, enquanto a absorção do metilmercúrio é de 90\%. Devido à elevada lipossolubilidade desta substância, esta tem afinidade com a mielina, onde inibe de forma eficaz a excitabilidade neuronal. O metilmercúrio pode interferir em fases críticas de desenvolvimento do sistema nervoso, como proliferação, migração, diferenciação, sinapse, mielinização a apoptose, provocando danos transitórios ou irreversíveis nas funções cognitivas, motoras e sensitivas. Os efeitos genotóxicos e teratogênicos da exposição intrauterina fetal ao metilmercúrio podem ser demonstrados por anomalias congênitas no sistema nervoso central (microcefalia e retardo mental importante). O metilmercúrio pode atravessar a barreira placentária e hematoencefálica, sendo que a sensibilidade fetal é de 3 a 4 vezes maior que a do adulto. Há outras formas de exposição infantil ao metilmercúrio como a amamentação e a sua ingestão com alimentos, água e mão contaminada. Ademais, o mercúrio pode conduzir a criança à insuficiência renal (Moniz \& Vollmer, 2017; Ayangbenro \& Babalola, 2017).

O chumbo é um metal tóxico pesado usado em construções, baterias, munições, plásticos, tintas, entre outros, e as formas mais comuns de sua absorção é através do consumo alimentar, água e ar, propiciando risco de exposição infantil pelas vias respiratória e gastrointestinal. Este xenobiótico gera efeitos tóxicos no sistema nervoso periférico e no sistema renal de adultos e no sistema nervoso central de crianças, bem como causa anemia hipocrômicamicrocítica, hipertensão, anorexia, redução da fertilidade, redução do crescimento físico e da estatura em crianças. Mesmo baixos níveis de exposição ao chumbo podem ser capazes de danificar o desenvolvimento neuropsicocognitivo infantil, devida relação dose-resposta não ser precisa. Além destes fatores, os efeitos de toxicidade do chumbo na infância são: redução da inteligência, desordens psicomotoras e neurocomportamentais, e podem ser permanentes (Ayangbenro \& Babalola, 2017; Moniz \& Vollmer, 2017). 


\section{Conclusão}

Os participantes dos últimos anos do curso de graduação em enfermagem avaliado não demonstraram conhecimento adequado sobre alguns fatores de exposição química infantil (uso de medicamentos na gestação; contato com cosméticos e estrogênio na infância; amamentação; dióxido de enxofre e metilmercúrio) indicando uma possível ausência de oferta de atividades de ensino sobre este tema no currículo em questão. Tal desconhecimento dos estudantes revela que a inclusão de conteúdos relativos à saúde ambiental infantil, especialmente da toxicologia, na formação do enfermeiro é fundamental, uma vez que, há diversos fatores químicos ambientais que colocam o feto, o neonato e a criança em risco a diversas doenças, transtornos e intoxicações. Este fato exige que os enfermeiros estejam preparados com competência para a aplicabilidade de ações de avaliação, vigilância e gerenciamento de riscos químicos.

Conclui-se que, há desafios para que profissionais da saúde, gestores e cientistas possam avançar e aplicar o conhecimento da toxicologia já adquirido em medidas de mitigação de fatores de exposição infantil, desde a gestação, aos poluentes ambientais e, assim, de prevenção de possíveis efeitos prejudiciais à saúde da criança em diferentes cenários da vida humana. Sugerem-se novos estudos avaliativos com graduandos de enfermagem e profissionais enfermeiros acerca da saúde ambiental infantil, de modo que, possam contribuir para revisão de estratégias de ensino, pesquisa e extensão e dos planos político-pedagógicos dos cursos de graduação em enfermagem no Brasil.

\section{Referências}

Ayangbenro, A. S., \& Babalola, O. O. (2017) A New Strategy for Heavy Metal Polluted Environments: A Review of Microbial Biosorbents. Int. J. Environ. Res. Public Health. 14(1): 1-16.

Bernardes, A. T. M. (2018) Medicação durante a gravidez. Dissertação de Mestrado. Faculdade de Medicina de Lisboa.

Borson, L. A. M. G., Martins, F. L., Comune, A. C., \& Guidi Junior, L. R. (2019) A exposição de substâncias na gestação e lactação. Revista Saúde em Foco. 11: 609-620.

Costa, A. L., Araujo Júnior, E., Lima, J. W., \& Costa, F. S. (2014) Fatores de risco materno associados à necessidade de unidade de terapia intensiva neonatal. Rev Bras Ginecol Obstet. 36(1): 29-34.

Ferreira, J. D., Couto, A. C., Alves, L. C., Oliveira, M. S. P., \& Koifman, S. (2012) Exposições ambientais e leucemias na infância no Brasil: uma análise exploratória de sua associação. R. bras. Est. Pop. 29(2): 477-492.

Finkler, M. G., Battisti, I. D. E., \& Anastácio, Z. F. C. (2019) Exposição de Crianças a Agrotóxicos: Uma Revisão Integrativa. INFAD Revista de Psicologia. $2: 147-156$.

García, A. C., Nieto, A. C., Hidalgo, P. L. P., Martos, S. S., \& Medina, I. M. L. (2018) Student nurses' knowledge and skills of children's environmental health: Instrument development and psychometric analysis using item response theory. Nurse Education Today. 69:113-119.

Gouveia, N., Leon, A. P., Junger, W., Lins, J. F., \& Freitas, C. U. (2019) Poluição do ar e impactos na saúde na Região Metropolitana de Belo Horizonte Minas Gerais, Brasil. Ciência \& Saúde Coletiva, 24(10):3773-3781.

Jan, C., Magoon, C., \& Ross, B. (2019) The Environmental Health of Children of Migrant Workers- an Example from China. Earth Systems and Environmental Sciences.

Maldaner, T. P., Santos, B. L., Weinert, L. V. C, \& Weinert, W. R. (2017) Saúde, sustentabilidade e infância: reflexões sobre uma perspectiva ambiental. Diversa Revista Eletrônica Interdisciplinar. 10(2): 60-68.

Mazoto, M. L., Filhote, M. I. F., Câmara, V. M., \& Asmus C. I. R. F. (2011) Saúde ambiental infantil: uma revisão de propostas e perspectivas. Cad. Saúde Coletiva. 19(1): 41-50.

Moniz, M. A., \& Vollmer, R. B. O. (2017) Exposure to environmental chemical contaminants and infant diseases: constant hazard? MOJ Toxicol. 3(4): $100-102$.

Moniz, M. A., Pereira, J. M., \& Marques, T. S. (2017) Tecnologia Educacional - Painel Integrado Saúde-Ambiente formação do enfermeiro: pesquisa descritiva. Online Braz. J. Nurs. 15(3): 466-471.

Moniz, M. A., Sabóia, V. M., Daher, D. V., \& Pereira, R. L. (2017) Práticas comunicativas socioambientais - Inovação e potencialidades no uso de tecnologias educacionais na formação do enfermeiro. Niterói: Eduff.

Moraes, S. L., Almendra, R., Santana, P., \& Galvani, E. (2019) Variáveis meteorológicas e poluição do ar e sua associação com internações respiratórias em crianças: estudo de caso em São Paulo, Brasil. Cad. Saúde Pública. 35(7):e00101418. 
Research, Society and Development, v. 10, n. 5, e18710514796, 2021

(CC BY 4.0) | ISSN 2525-3409 | DOI: http://dx.doi.org/10.33448/rsd-v10i5.14796

Nascimento, S. N. (2017) Potenciais Efeitos Tóxicos de Xenobióticos Ambientais Sobre a Saúde Infantil no Meio Rural. Porto Alegre: Universidade Federal do Rio Grande do Sul.

Paiva, R. F. P. S., \& Souza, M. F. P. (2018) Associação entre condições socioeconômicas, sanitárias e de atenção básica e a morbidade hospitalar por doenças de veiculação hídrica no Brasil. Cad. Saúde Pública. 34(1): e00017316.

Pereira, G., \& Ortegão, M. I. R. (2016) Pesquisa quantitativa em educação: algumas considerações. Periferia - Educação, cultura e comunicação. 8(1): 66-79.

Perlroth, N. H. \& Branco, C. W. C. (2017) Current knowledge of environmental exposure in children during the sensitive developmental periods. J. Pediatr. 93(1): 17-27.

Rocha, P. C., Alves, M. T. S. S. B., Chagas, D. C., Silva, A. A. M., Batista, R. F. L., \& Silva, R. A. (2016) Prevalência e fatores associados ao uso de drogas ilícitas em gestantes da coorte BRISA. Cad. Saúde Pública. 32(1) e00192714.

Sousa J. S., Campos, R. T., Silva, A. F., Bezerra F. N. R., \& Lira J. S. (2016) Estimação e análise dos fatores determinantes da redução da taxa de mortalidade infantil no brasil. Revista Brasileira de Estudos Regionais e Urbanos. 10(2): 140-155.

World Health Organization. (2017) Inheriting a sustainable world? Atlas on children's health and the environment. WHO.

Zendron, R. (2015) Mecanismos de neurotoxicidade e doenças neurológicas relacionadas à intoxicação por metais pesados. Revista Brasileira de Nutrição Funcional. 15(64): 45-51 